\title{
Coronal waves in coronal loops during non-flare stage
}

\author{
Dmitry Prosovetsky \\ The Institute of solar-terrestrial physics, Lermontov st., 126a, \\ p/o box 291, Irkutsk, 664033, Russia \\ email: proso@iszf.irk.ru
}

\begin{abstract}
Using SOHO/EIT Fe XII $\lambda 195 \AA$ observations the new type of oscillations in coronal loops was detected. The oscillation corresponds to wave propagated to outer area of atmosphere of active area. As opposed to most kind of oscillations associated with coronal loops the waves are observed at non-flare stage of active areas evolution. Velocities of the wave propagation were 8$20 \mathrm{~km} \mathrm{~s}^{-1}$ and had quasi-perpendicular direction with magnetic field. Such waves were detected in active areas located on solar disk and loops structures outside solar limb. Investigation of EIT data shows the waves are not result of changes of topology of a magnetic field and loops configuration. The nature and probable sources of waves are discussed.
\end{abstract}

Keywords. Sun: activity, UV radiation, oscillations

\section{Introduction}

The oscillations of coronal structures are typical process in solar corona. This phenomenon is observed in wide range of time and spatial scales, any topology of magnetic field. Oscillation is special interesting in solar active regions. There oscillation can be expressed as source of released energy or as their response. In the atmosphere of active regions the oscillations correspond with process of propagation, redistribution and release of energy.

Observations of oscillations on higher atmospheric levels are hard in view of weak emission. Observations become too easy during and after flare events when atmosphere emission increased. For example De Moortel \& Brady (2007) detected the higher harmonic loop oscillation after powerful flare. Raouafi et al. (2004) investigated shock wave associated with expanding loop system. Such large-scale events as EIT wave connect to flares or CME (e.g. Chertok \& Grechnev (2003), Biesecker et al. (2002), Wills-Davey et al. (2007)).

Quiet events are more difficult for observation. Special observation programs and data processing methods are necessary for their detection. Special program of TRACE observation made possible by De Groof et al. (2004) to detect and investigate propagating disturbances in loop. Tomczyk et al. (2007) reported about very interesting observation of oscillation of velocities field in quiet system of loop using Coronal Multichannel Polarimeter.

\section{Observations}

However, today most available data are observations of SOHO/EIT and TRACE. These data are irreplaceable for regular investigations of coronal oscillation. Coronal wave as result this research was detected investigating EIT data.

For demonstration of phenomenon EIT data are chosen EIT data on December 13, 2006. AR 10930 (Fig. 1a) where oscillation seen most clearly was investigated. This active 


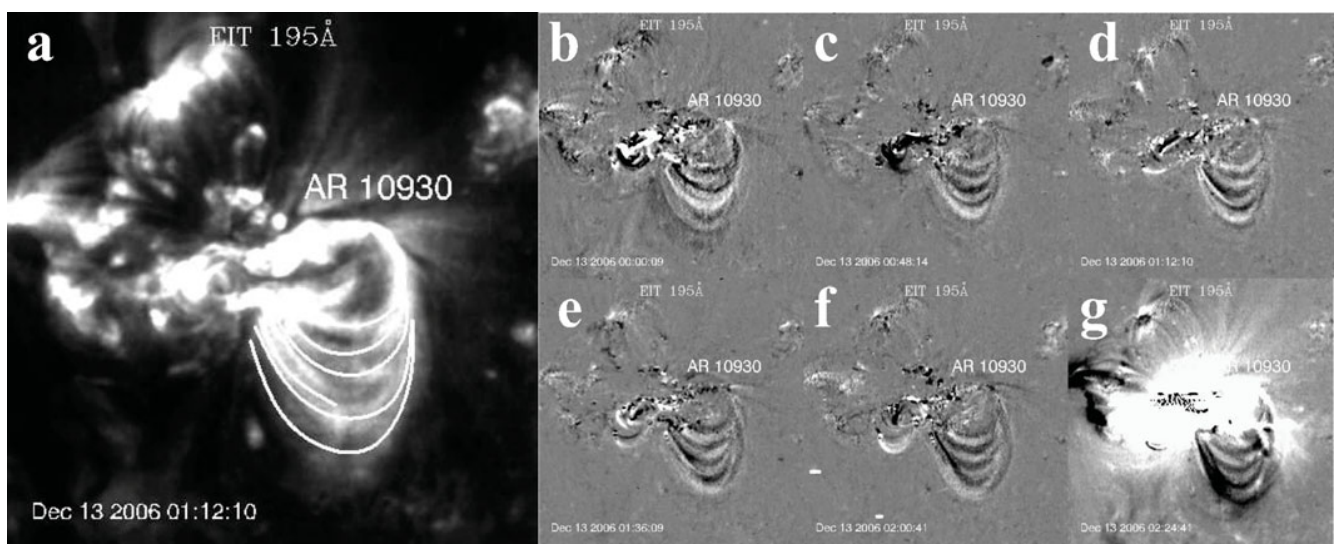

Figure 1. Propagation of wave in the atmosphere of active region NOAA 10930 at Dec 13,

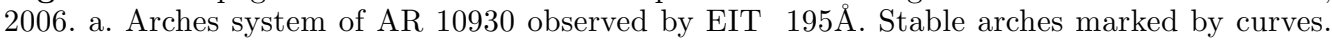
b.-g. Allocation of wave train from 00:00:09 to 02:24:41 UT on the difference images.

region produced series of powerful flares and CME events. At December 13 02:14 UT the flare was registered with GOES X-ray class X3.4. At 2:54 UT was detected large coronal mass ejection event in west-south quadrant. All EIT images were rotated to the some equal time before flares. Active region was cut out and frames prior to flare were processed. Unfortunately such frames were only twelve because EIT was "bake out". Before flare we can see the system of stationary arches. Several bright arches were marked (Fig. 1a).

Oscillation was contrasted by special method. The differences between neighboring frames were found for each pixel using formula $I_{i j}^{\prime}\left(t_{k}^{\prime}\right)=I_{i j}\left(t_{k+1}\right)-I_{i j}\left(t_{k}\right)$. This method of preparation is used usually for allocation fast changing elements of solar images. Fig. 1b-g shows the plasma in arches system is not stationary. In west south part of active region we can see clearly visible waves propagating out from base of active region. There were three or four waves in different time with periodically structure in direction outward from the Sun. In radial direction there is the sequence of waves which starts on height about five thousand kilometers above photosphere. Waves are not seen on EIT images where there are only stationary loops.

The time cadence of used observations could be increased using TRACE observation. Unfortunately the wavetrain aren't seen on TRACE differences sequences. The start of TRACE observation was at 1:47 UT. EIT difference images show the loop appreciably changed between this time and start of flare. Outside flare time on TRACE data is visible only noise. It means the TRACE data are not useful for study quiet events.

The event at December 13 investigation shows the wave is not being detected at levels above EIT arches system. It can be simply explained. First: upper levels of arches system are visible on the quiet sun background. Second, probably the wave's amplitude decreases in low density plasma. On Fig. 2 is shown EIT images and differences sequence at December, 17 2006. Active region 10930 is allocated at west solar limb now. On EIT data there is CME event at 15:30 pm. Before CME magnetic loops were erupted during 30 minutes.

On Fig. 2a there is the stationary loop which stay stable several hours. Wave train with sharp wave front can be found on difference pictures (e.g. Fig. 2b-d) during of 12 hours. Parameters of waves in this day of observation are similar to December, 13. 


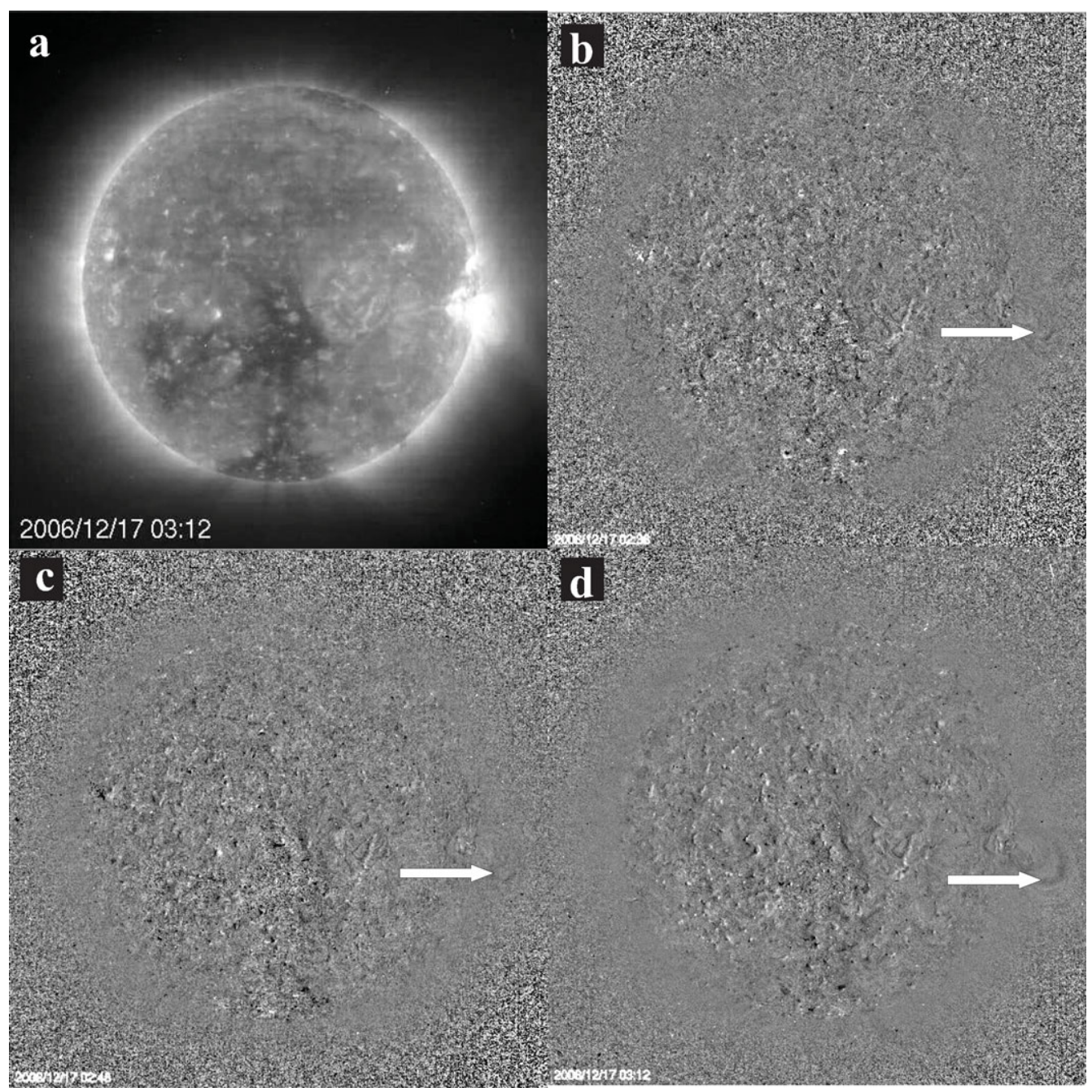

Figure 2. The wave train outside of solar limb at Dec 17, 2006. a. Solar disk according on data of EIT Fe XII 195Åline. b.-d. Allocation of wave front at 02:36 UT (b), 02:48 UT (c) and 03:12 UT (d).

The waves are being detected not so well at as at Dec. 13. It is easy to explain if one takes into account the values of plasma density at coronal level.

\section{Discussion}

For nature of observed waves explanation, it is important to know the plasma parameters. It is not so simple. But the density can be taken from EIT data. Brosius et al. (2002) compared EIT intensity and density taken from Fe XV line data. They found simple dependence $\lg N_{e, i}=8.34+0.509 \lg I_{E I T}$, where $N_{e, i} \geqslant 2.2 \cdot 10^{8}$. This dependence may help obtain density from EIT intensity range in arches.

In Table 1 is collected some properties of waves events which were observed by SOHO during 2006. Except the wave's properties in Table 1 the information about flares and $\mathrm{CME}$ is showed. As we can see the waves velocities was very slow. In Table $1 \mathrm{~T}$ and $\mathrm{L}$ 
Table 1. Some properties of waves.

\begin{tabular}{|c|c|c|c|c|c|c|c|c|c|}
\hline $\begin{array}{l}\text { Date \& time, } \\
\text { start, end, UT. }\end{array}$ & $\begin{array}{l}\text { Coord., } \\
\text { NOAA No }\end{array}$ & $\begin{array}{c}\mathbf{V} \\
\mathbf{k m ~} s^{-1}\end{array}$ & $\mathrm{~T}$ & $\begin{array}{l}\mathrm{L}, \\
\mathrm{km}\end{array}$ & $\begin{array}{c}\mathbf{N}_{e, i}, \\
\times \mathbf{1 0}^{9} \mathbf{c m}^{-3}\end{array}$ & $\begin{array}{c}\Delta \mathbf{N}_{e, i}, \\
\times \mathbf{1 0}^{9} \mathbf{c m}^{-3}\end{array}$ & CME & Flare & $\begin{array}{l}\text { GOES } \\
\text { Class }\end{array}$ \\
\hline $\begin{array}{l}2006 / 12 / 12 ? \\
-12 / 1302: 24\end{array}$ & $\begin{array}{c}\text { S05W } 23 \\
10930\end{array}$ & $8-20$ & $\begin{array}{l}750- \\
1900\end{array}$ & 15000 & 4.2 & 1.2 & $02: 54: 04$ & 02:40:00 & X 3.4 \\
\hline $\begin{array}{l}2006 / 12 / 14 \\
0: 59-02: 53\end{array}$ & $\begin{array}{c}\text { S06W } 46 \\
10930\end{array}$ & 20 & 600 & 12000 & 3.6 & 0.8 & $20: 30: 04$ & $22: 15: 00$ & $\mathrm{X} 1.5$ \\
\hline $\begin{array}{l}2006 / 07 / 06 \\
05: 36-07: 12\end{array}$ & $\begin{array}{l}\text { S09E } 38 \\
10875\end{array}$ & 15 & 670 & 10000 & 2.5 & 0.9 & 08:54:00 & $08: 36: 00$ & M2.5 \\
\hline $\begin{array}{l}2006 / 04 / 27 \\
9: 53-12: 44\end{array}$ & $\begin{array}{l}\text { S08W } 54 \\
10865\end{array}$ & 10 & 1400 & 14000 & 3.9 & 0.8 & - & $15: 52: 00$ & M 7.9 \\
\hline $\begin{array}{l}2006 / 04 / 26 \\
12: 12-15: 24\end{array}$ & $\begin{array}{l}\text { S09W } 35 \\
10898\end{array}$ & 9 & 1100 & 10000 & 4.7 & 0.9 & - & $17: 02: 00$ & M1.3 \\
\hline $\begin{array}{l}2006 / 04 / 06 \\
9: 24-12: 44\end{array}$ & $\begin{array}{l}\text { S08W 61 } \\
10875\end{array}$ & 18 & 610 & 11000 & 3.1 & 1.0 & - & $\begin{array}{l}20: 34: 00 \\
05: 35: 00\end{array}$ & $\begin{array}{l}\text { M1.3 } \\
\text { M1.4 }\end{array}$ \\
\hline $\begin{array}{l}2006 / 12 / 17 \\
2: 34-14: 44\end{array}$ & outlimb & $9-15$ & $\begin{array}{l}750- \\
1000\end{array}$ & 15000 & 0.4 & 0.2 & $15: 30: 04$ & $22: 15: 00$ & $\mathrm{C} 2.1$ \\
\hline
\end{tabular}

are period and wavelength, the plasma density are typical for hot coronal arches and differences between low and high value of density in the waves is labeled as $\Delta N_{e, i}$.

On Fig. 3 some angular and radial properties of waves are shown. From center of arches system base via equal angle depend values of density and velocity. On Fig. 2a for one of waves is shown value of velocity for time of observation. It is seen in some time velocity changed for all angles when wave position corresponded to position of stable arches. In other hand density of waves maxima weakly changes with distance from base of arches system. On Fig. 2b angular dependence for different waves in the same time is shown. Similar properties there are in all of wave's observation.

Thereby the wave always propagates in quasi-perpendicular direction to magnetic field. Properties of wave depend on plasma density and magnetic field value. The value of coronal magnetic field can be found roughly from dipole approximation. For example
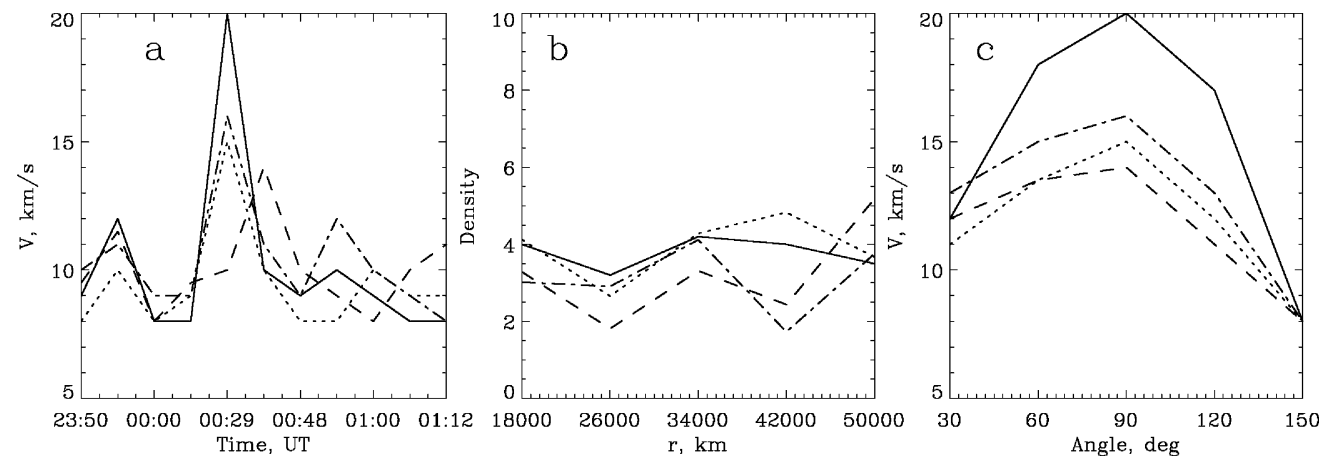

Figure 3. Angular, spacing and time dependence of wave velocities and plasma density. a. Angular and time distribution of leading front of wave train at Dec. 13, 2006. Each line corresponds to value of angle with interval of 30 degree between geometric center of loops system and perpendicular to magnetic field b. Dependence between distance from photosphere and measured plasma density for the same angles c. Angular dependence of velocities for different waves on Dec 13, 2006 
at Dec. $13 B(R)=B_{\text {foot }}\left(1+\frac{R}{R_{D}}\right)^{-3} \approx 60 G$ where $B_{\text {foot }}$ the magnetic field at base of dipole, and $R_{D}$ the dipole radius. Then the Alfvén speed $v_{A}=\frac{B}{\sqrt{4 \pi \rho}} \approx 2000 \mathrm{~km} \mathrm{~s}^{-1}$. The densities were determined, temperature forming Fe XII $195 \AA$ line is 1.5 million Kelvin. Unfortunately, magnetic field is known only at photospheric level. In this case sound velocity $c_{s}^{2}=\frac{2 \gamma k_{B} T}{\mu m_{p}} \approx 180 \mathrm{~km} \mathrm{~s}^{-1} \leqslant v_{A}$. Such slow velocity and quasi-perpendicular propagation to magnetic field allow assuming the waves can be acoustic waves with dependence from magnetic field. That is magneto acoustic waves.

The velocity for two fast and slow modes is depending from magnetic field, density and temperature $v_{f, s}^{2}=\frac{1}{2}\left(v_{A}^{2}+c_{s}^{2} \pm \sqrt{v_{A}^{4}+c_{s}^{4}-2 v_{A}^{2} c_{s}^{2} \cos 2 \theta}\right)$. For depended values the velocity of slow magneto acoustic waves there is realistic values.

In conclusion I note such events may transfer the energy to the upper levels. It is necessary to take this event into account for development the models of solar atmosphere. I hope unusual phenomenon will investigated fully henceforward.

\section{Acknowledgements}

I express sincere gratitude to SOHO/EIT team for data used in this research. The work was supported by RFBR grant No 08-02-08680 and the Program of Presidium RAS No 13.

\section{References}

Biesecker, D. A., et al. 2002, ApJ, 569, 1009

Brosius, J. W., et al. 2002, ApJ, 574, 453

Cadez, V. M. \& Ballester, J. L. 1994, ApJ, 292, 669

Chertok, I. M. \& Grechnev, V. V. 2003, Astron. Rep., v. 47, 11, 934

De Groof, A., Berghmans, D., van Driel-Gesztelyi, L., \& Poedts, S. 2004, A\&SA, 415, 1141

Delaboudiniere, J.-P., Artzner, G. E., Brunaud, J., et al. 2004, Solar Phys., 162, 1-2, 291

De Moortel, I. \& Brady, C. S. 2007, ApJ, v. 664, 2, 1210

O'Shea, E., Srivastava, A. K., Doyle, J. G., \& Banerjee, D. 2007, A\&A, v. 473, 2, L13

Pascoe, D. J., Nakariakov, V. M., \& Arber, T. D. 2007, A\& A, 461, 1149

Raouafi, N.-E., et al. 2004, A\& $A, 424,1039$

Tomczyk, S., et al. 2007, Science, v. 317, 5842, 1192

Wills-Davey, M. J., DeForest, C. E., \& Stenflo, J. O. 2007, ApJ, 664, 556 